\title{
Paper
}

\section{Dual-polarized Ultra-wideband Antenna with Improved Polarization Purity}

\author{
Rabia Yahya ${ }^{\dagger}$, Akira Nakamura $^{\dagger}$ (member), Makoto Itami $^{\dagger}$ (member) and Tayeb A. Denidni ${ }^{\dagger \dagger}$
}

\begin{abstract}
In this paper, a compact UWB dual-polarized antenna with improved co-to-cross-polarization ratio is proposed. This antenna is fed by two orthogonal tapered CPW lines to achieve dual-polarization operation. To enhance the polarization purity and the isolation level between the antenna's ports, a new technique is proposed. The detailed design, as well as the analysis of the obtained results are presented and discussed, which revealed that the proposed antenna is matched over the entire UWB band with good isolation level between its ports of $20 \mathrm{~dB}$ and high co-to-cross polarization ratio of more than $20 \mathrm{~dB}$. With these features, the proposed antenna is an optimized candidate for media applications.
\end{abstract}

Keywords: UWB antennas, diversity applications, polarization purity.

\section{Introduction}

To satisfy the growing requirements of today media applications, the quantity and quality of the provided services must be optimized, which can be achieved through improving the data rate and enhancing the communication channels capacity. Therefore, it is convenient to propose using technologies that can provide high data rate and wise utilization of the communication channels. UWB technology along with the diversity techniques can satisfy those requirements at the same time. UWB technology has gained wide interest since the release of the FCC, in 2002, which allows the use of the band from 3.1 to $10.6 \mathrm{GHz}$ without license for the UWB applications ${ }^{1)}$. This wide band allows the transfer of pulses with a high speed, which can provide a high data rate and thus interference mitigation. As a result, UWB technology has become one of the most developed and promising technologies and the first choice for some applications such as wireless personal area networks (WPAN) ${ }^{2}$. On the other hand, the diversity techniques have been proposed to optimize the capacity of the communication systems and improve the quality of the transmission by reducing the fading effect caused by interference and multipath phenomena as demonstrated by several studies ${ }^{344)}$. One of the best

\footnotetext{
Received April 5, 2016; Revised May 16, 2016; Accepted June 14, 2016

$\dagger$ Department of Applied Electronics, Tokyo University of Scienc (Tokyo, Japan)

† Institut National de la recherch_Scientifique (INRS)

(Montreal, Canada)
}

diversity techniques is the polarization diversity, which enables the increase of the system capacity by caring separate information on orthogonal polarizations over the same physical link at the same frequency. Therefore, ultra-wideband dual-polarized technology is of great interest for short range communications, such as media applications. Much research has been done to develop ultra-wideband antennas with dual-polarization operation ${ }^{5)-8)}$. The proposed antennas suffer from several problems such as a narrow bandwidth ${ }^{5)}$, which is not large enough to cover the entire UWB band; bulky forms ${ }^{6}$, with complicated feeding structures require more than one substrate, which increases the overall cost of the antenna ${ }^{7) 8}$. The problem of low isolation level between the used ports to achieve dual-polarization operation has been studied $\mathrm{in}^{2}$. $\mathrm{In}^{9)}$, an UWB dualpolarized antenna based on microstrip technology has been proposed. However, compared to microstrip technology, CPW (coplanar waveguide) technology has superior advantages regarding dispersion, radiation loss and easy integration with monolithic microwave integrated circuits. Furthermore, CPW-fed slot structures printed on one side of the substrate allow to avoid the misalignment errors, which is critical in microstrip-fed structures ${ }^{10)}$.

The high cross-polarization level may deteriorate the radiation characteristics of the antenna, especially those with dual-orthogonal-polarization operation, which need to have quite good polarization purity. Some applications require precise co-to-cross polarization level to enable 
certain operations such as the avoidance of the crosstalk in the communication channels. Therefore, several techniques have been proposed to overcome the problem of high cross-polarization level ${ }^{11) 12)}$. In $^{11)}$, two substrates have been used to enhance the polarization purity of conventional tapered slot antenna, and this technique has also been used by some dual-polarized antennas, but it increases the overall cost of the antenna by using multiple substrates instead of one. $\mathrm{In}^{12)}$, differential feeding technique has been used, where a single polarization is radiated through two oppositely lying ports and dual-polarization by four ports, which require the employment of out-of-phase power divider, operating at each frequency of the interested band to feed the antenna's ports by signals equal in amplitude and outof-phase. Therefore, to overcome this problem, we first propose the design of a compact ultra-wideband dualpolarized antenna based on CPW technology. After that, we propose a technique to enhance the isolation level across the entire UWB band while keeping the ability of the antenna to radiate over the same band with two orthogonal polarizations. The effect of this technique on the cross-polarization level is subsequently studied, and its negative influence is corrected to obtain good isolation and low cross-polarization level at the same time. The details of the proposed antenna design and the proposed techniques are presented and discussed in the next sections. It worth to mention that the analysis and optimization were performed using CST MICROWAVE STUDIO 2015.

\section{Antenna design}

The proposed antenna is a circular slot cut of a ground plane printed on substrate $\mathrm{RO} 4003 \mathrm{C}$ of dielectric constant of 3.38 , loss tangent of 0.0027 and dimensions of $(72 \mathrm{~mm} \times 72 \mathrm{~mm})$, this way, a dipole-like radiation can be obtained. The circular slot is fed by two orthogonal $50 \Omega$ CPW tapered lines through two semi-discs, placed in an orthogonal manner to achieve dual-polarization operation, as shown in Fig.1.a. The impedance matching band of this structure is governed by several parameters such as the dimensions of the circular slot and the semicircular discs as well as those of the CPW lines that are tapered to increase the matching band, especially at high frequencies. All these parameters were optimized to enable an operating band from 3.1 to $10.6 \mathrm{GHz}$ with reflection coefficient inferior to $-10 \mathrm{~dB}$, as shown in Fig.2. However, the isolation between the antenna ports is inferior to $15 \mathrm{~dB}$, which is not sufficient for the diversity

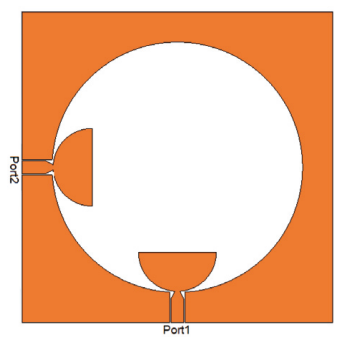

(a) UWB dual-polarized antenna

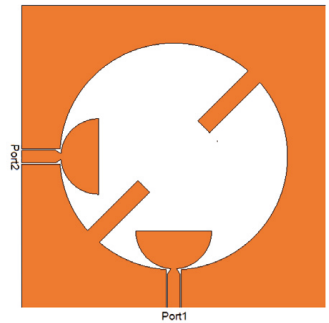

(b) With isolation technique

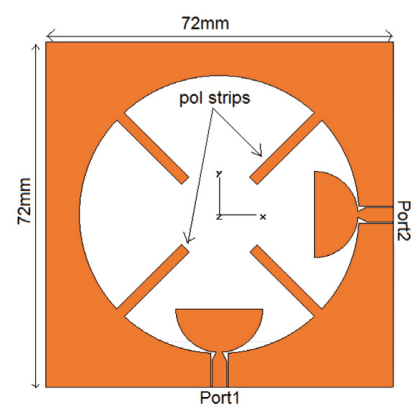

(c) Structure of the proposed antenna

Fig. 1 Steps of designing the proposed antenna.

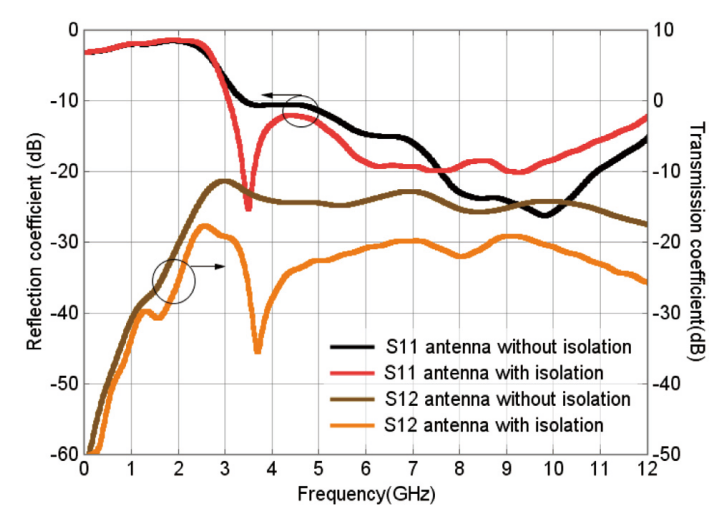

Fig. 2 S-parameters of the structure 1.a with and without the isolation.

requirements. Therefore, it needs to be improved. To accomplish so, two strips are inserted diagonally around the center of the circular slot as shown in Fig.1.b. As a result, the isolation level is enhanced while keeping an UWB operating band as also indicated in Fig.2. The insertion of the isolation strips modifies the current distribution on the structure and minimizes the mutual coupling between the two ports by intercepting the radiation coming from the excited port. Hence, a high isolation level is reached. In meanwhile, the radiation of the inserted strips affects the polarization purity of the antenna and leads to high cross-polarization, as indicated in Fig.3, where the radiation patterns of the antenna with and without the isolation structure, at $7 \mathrm{GHz}$, are shown. Therefore, we propose a new technique to minimize the effect of the isolation structure on the cross-polarization while keeping the 


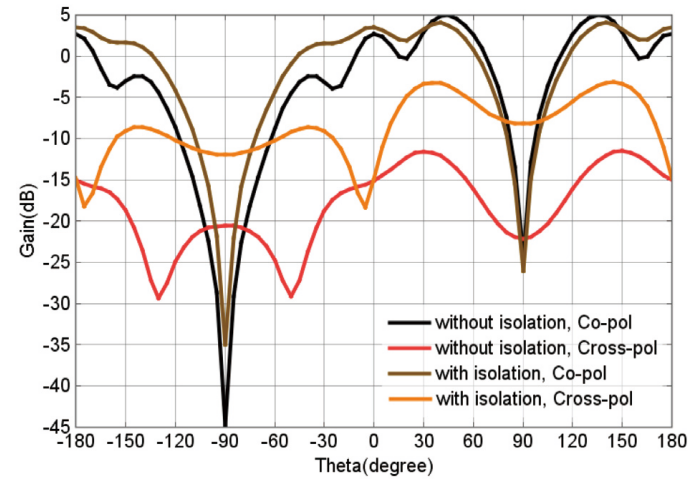

Fig. 3 Radiation patterns of the antenna with and without the isolation at $7 \mathrm{GHz}$.

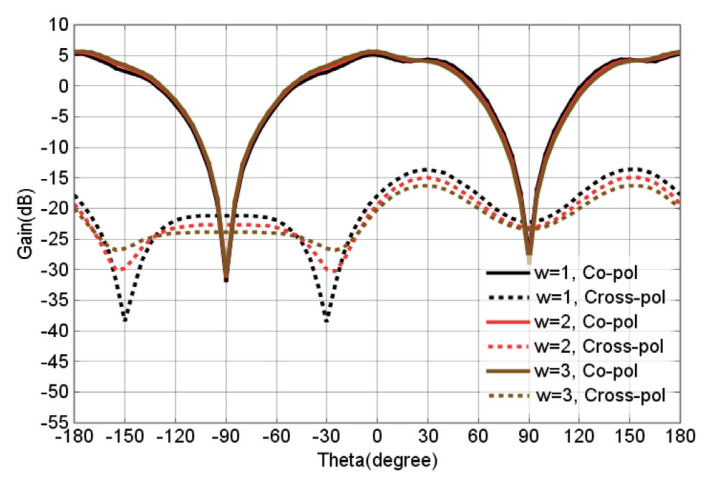

Fig. 4 Co and Cross-polarized gain over Theta at $7 \mathrm{GHz}$ for different values of $\mathrm{w}$.

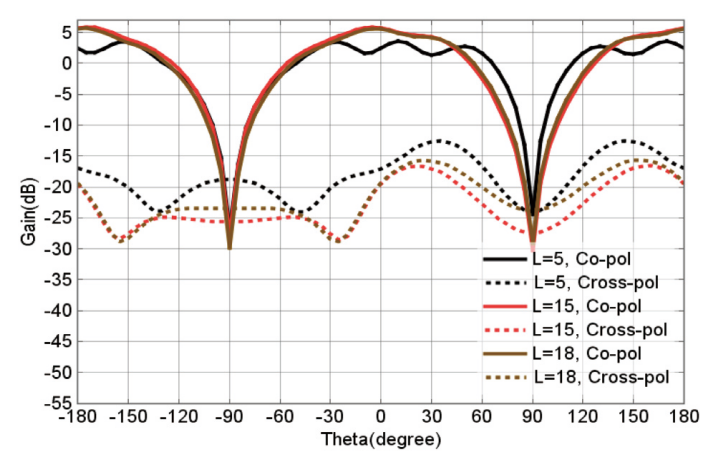

Fig. $5 \mathrm{Co}$ and Cross-polarized gain over Theta at $7 \mathrm{GHz}$ for different values of $\mathrm{L}$.

high isolation level achieved and the operating bandwidth. This technique consists of inserting two diagonally strips (pol-strips) in the circular slot as illustrated in Fig.1.c; these strips minimize the effect of the isolation structure on the cross-polarization level and enhance the isolation.

To study the influence of the inserted strips on the radiation performance of the antenna, the co-polarized gain and cross-polarized gain, obtained at the center of the UWB band, over azimuth angle Theta, for different dimensions of the pol-strips, were numerically
Table. 1 Optimized values of the proposed antenna dimensions.

\begin{tabular}{|l|l|l|l|l|l|}
\hline Rs & Rp & wm & g & L & w \\
\hline $29 \mathrm{~mm}$ & $9 \mathrm{~mm}$ & $3 \mathrm{~mm}$ & $0.3 \mathrm{~mm}$ & $19 \mathrm{~mm}$ & $2.2 \mathrm{~mm}$ \\
\hline
\end{tabular}

computed, and they are shown in Fig.4 and Fig.5; where $\mathrm{w}$ and $\mathrm{L}$ denote, respectively, the width and the length of pol-strips. Fig. 4 was obtained for $\mathrm{L}=19 \mathrm{~mm}$ and Fig. 5 was obtained for $w=2 \mathrm{~mm}$. It can be observed that varying the width and the length of the strips affects the cross-polarization level in different ways. In the interval of Theta $>0$, the cross-polarized gain is inversely proportional to the strips width. In the case of swiping the length of the strips, the cross-polarized gain varies in a non-linear manner. Although the co-polarized gain, when the strips length is varying, shows weak variation especially around theta $=0$, its variation is stronger than the results from varying the strips width. Hence, the copolarized gain and the cross-polarized gain over Theta in the E-plane depend highly on the strips dimensions. Therefore, the cross-polarization level can be controlled by the dimensions of the strips. Both of the gains give best cross-polarization level for strips dimensions similar to those used for the isolation. Hence, the polstrips act as a canceler of the isolation strips effect on the cross-polarization level. In order to find the dimensions that provide the best performance, in terms of the operating bandwidth, the isolation between the two ports and the cross-polarization level, the overall structure was optimized. Table.1 illustrates those dimensions that give the best performance; where $\mathrm{Rs}$ is the radius of the circular slot, $\mathrm{Rp}$ is the radius of the semi-circular patch, wm is the width of CPW lines metal strip, and $\mathrm{g}$ is the gap between the strip and the coplanar ground plane. The results of the proposed antenna, with the optimized dimensions, that prove its performance are described and discussed in the next section.

\section{Results and discussion}

The proposed antenna was fabricated and its photograph is shown in Fig.6. The S-parameters simulated and measured, at Port1, are plotted in Fig.7. As indicated, the input reflection coefficient $\mathrm{S}_{11}$ is inferior to $-10 \mathrm{~dB}$ over the entire UWB band and the transmission coefficient $\mathrm{S}_{21}$, signifying the isolation level between the two ports, has a worst value of $-17 \mathrm{~dB}$ and an average value of $-20 \mathrm{~dB}$ across the UWB band, which satisfies the isolation level required for diversity 


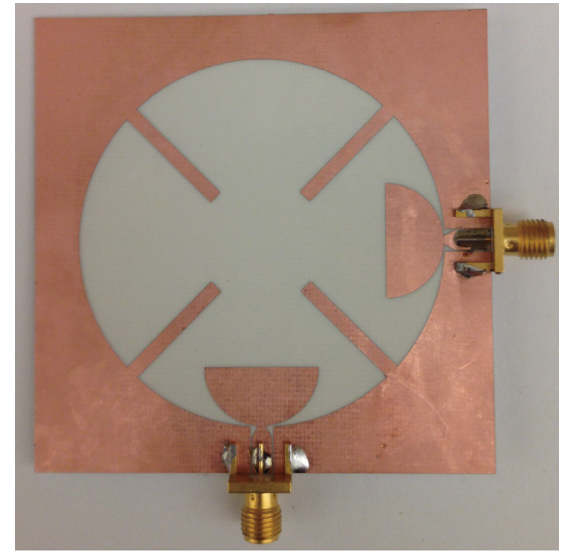

Fig. 6 Photograph of the fabricated prototype.

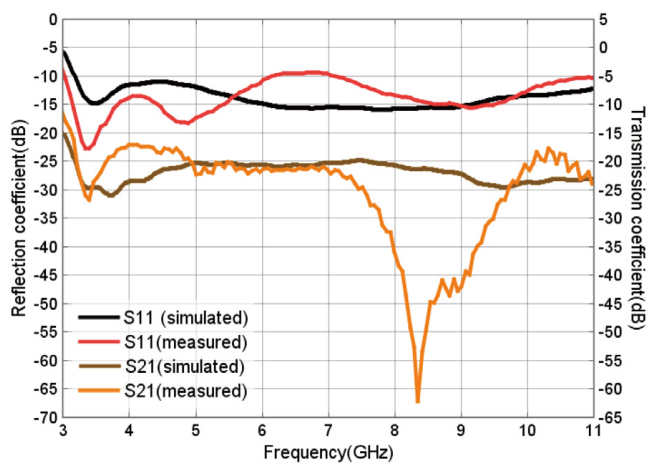

Fig. 7 S-parameters of the proposed antenna.

communication systems. In addition, a good agreement between the simulation and measurement results can be observed. It should be mentioned that, as a result of the symmetry and similarity of the antenna structure, the S-parameters obtained at the Port1 and those obtained at the Port2 are similar, and the radiation patterns are also similar with a rotation of 90 degrees. Therefore, only those simulated and measured at the Port1 are presented in this paper.

To visualize the radiation performance of the proposed antenna, the co-polarized gain and cross-polarized gain simulated at Port1 over different values of azimuth angle Theta in the E-plane at the frequencies $3 \mathrm{GHz}$, $7 \mathrm{GHz}$, and $10 \mathrm{GHz}$, which correspond to the lower, central and upper frequencies of the UWB band, are shown in Fig.8. From these curves, it can be noticed that the gain changes according to the value of angle Theta and also varies as a function of the frequency. While the maximum radiation is in $\mathrm{Z}$-direction (Theta $=0$ ) at $7 \mathrm{GHz}$, it is aligned at $3 \mathrm{GHz}$ and $10 \mathrm{GHz}$. In order, to validate the concept of the proposed technique to minimize the cross-polarization level and to prove its effectiveness, the co-polarized gain and cross-polarized gain of the

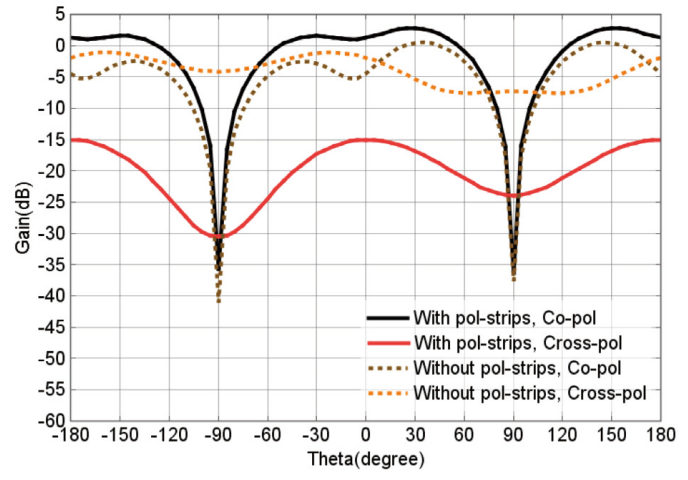

(a) $3 \mathrm{GHz}$

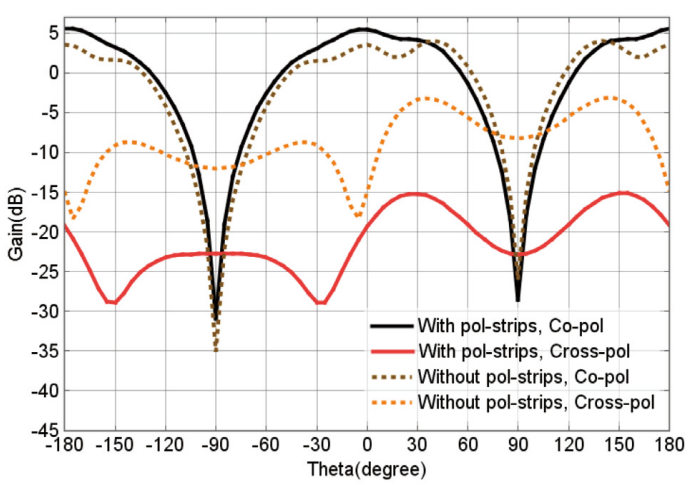

(b) $7 \mathrm{GHz}$

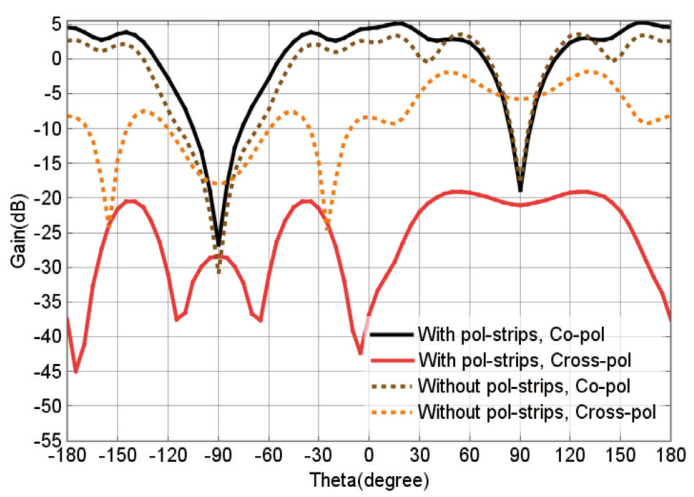

(c) $10 \mathrm{GHz}$

Fig. 8 Co and Cross-polarized gain over theta in E-plane with and without pol-strips.

proposed structure without pol-strips are also shown in Fig.8. The comparison between the co- and crosspolarized gains of the antenna with and without polstrips reveals that in addition to the ability of the polstrips to reduce the cross-polarized gain, they are also capable of enhancing the co-polarized gain and significantly reducing the cross-polarization level. Comparing with the antenna without pol-strips, the cross-polarization level of the proposed antenna (antenna with pol-strips) is reduced by more than $15 \mathrm{~dB}$ across the UWB band. Based on these results, we can say that the insertion of the pol-strips enhances the polarization purity of the antenna by overcoming the effect of the isolation strips radiation and improves the 


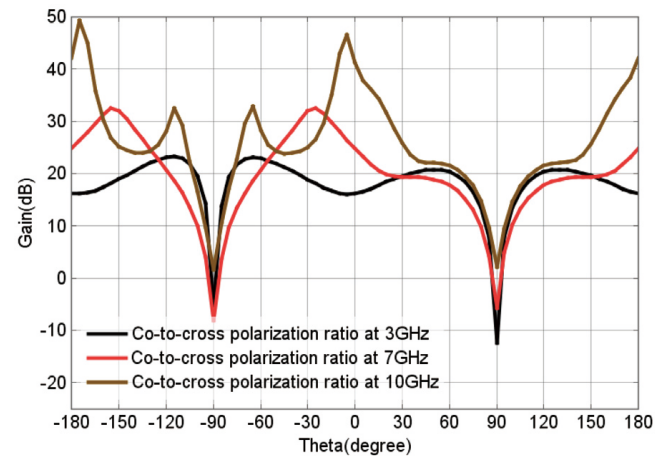

Fig. 9 Co-to-cross polarization ratio at different frequencies.

isolation between the two ports. Finally, the Co-to-cross polarization ratio of the proposed antenna, at different frequencies of the UWB band, is shown in Fig.9. As indicated, the co-to-cross polarization ratio is more than $20 \mathrm{~dB}$ around the angles at which the maximum radiation occurs and its worst values are at the angles $-90^{\circ}$ and $90^{\circ}$, where the radiation nulls occur.

\section{Conclusion}

Easy fabrication, compact size and low profile are some of the attractive merits that increase the demand of planar antennas for different applications. Therefore, recently, many planar antennas have been proposed for ultra-wideband systems with diversity techniques. The diversity techniques can maximize the capacity of UWB systems and improve their services. However, the proposed antennas for these applications suffer from several problems, such as narrow bandwidth, large size, low isolation level and high cross-polarization. The proposed antenna aims to overcome these problems with simple and effective techniques can also be used by other structures to increase their diversity performance as well as their radiation performance by enhancing the isolation and cross-polarization levels. The proposed antenna is compact size ultra-wideband antenna with an overall size of $(72 \mathrm{~mm} \times 72 \mathrm{~mm})$ printed on one side of the substrate using CPW technology. The details of its design have been described, analyzed and discussed. The results prove the ability of this antenna to operate over the band $3.1-10.6 \mathrm{GHz}$ with a high isolation level between its two ports. It can radiate through two orthogonal linear polarizations providing high polarization purity across the entire UWB band which has been achieved by using new techniques to minimize the cross-polarization level and the coupling effect between the used orthogonal ports. The performance of the proposed antenna and its features make it a good candidate for UWB communication systems with diversity operation as media applications.

\section{References}

1) First Report and Order, Revision of Part 15 of the Commission's Rules Regarding Ultra-Wideband Transmission Systems, Federal Communications Commission, ET Docket 98-153, FCC 02-48 (Feb. 2002)

2) C. Yunlong and Z. Feng: "A Dual-polarized printed UWB antenna", Microwave and Optical technology letters. 51, 5, pp.1177-1180 (May 2009)

3) J.H. Winters, J. Salz and R.D. Gitlin: "The impact of antenna diversity on the capacity of wireless communication systems", IEEE trans. Communications. 42, 2/3/4, pp.1740-1751 (Feb. /Mar. /Apr. 1994)

4) R.G. Vaughan, J.B. Andersen: "Antenna diversity in mobile communications", IEEE trans. Vehicular technology. vol. VT-36, No.4, pp.149-172 (Nov. 1987)

5) Chi-Hsuan Lee., Shih-Yuan Chen. and Powen Hsu: "Isosceles triangular slot antenna for broadband dual polarization applications", IEEE trans. Antennas and Propag. 57, 10, pp.33473351 (Oct. 2009)

6) A. Grzegorz, T. ZWICK and W. Werner: "Compact, dual-polarized UWB-antenna, embedded in a dielectric", IEEE trans. Antennas and Propag. 58, 2, p. 279-286 (2010)

7) S.K. Padhi, N.C. Karmakar, Sr.,C.L. Law and S. Aditya, Sr: "A Dual Polarized Aperture Coupled Circular Patch Antenna Using a C-Shaped Coupling Slot", IEEE trans. Antennas and Propag. 51, 12, pp.3295-3298 (Dec. 2003)

8) M. Barba: "A High-Isolation, Wideband and Dual-Linear Polarization Patch Antenna", IEEE trans. Antennas and Propag. 56, 5, pp.1472-1476 (May 2008)

9) A. Daviu, E. Gallo, M. cabedo-Fabrés, M. Ferrando-Bataller, M: "Novel ultra-wideband antenna for diversity applications", 2010 Antennas and Propagation Society International Symposium (APSURSI) IEEE 2010, pp.1-4 (July 2010)

10) F. Consoli, F. Maimone and S. Barbarino: "Study of a CPW-Fed Circular slot antenna for UWB communications", Microwave and Optical technology letters. 48, 11, pp.2272-2277 (Nov. 2006)

11) O. Majid, S. Noghanian and L. Shafai: "A modified double layer tapered slot antenna with improved cross polarization", 13th International Symposium on Antenna Technology and Applied Electromagnetics and the Canadian Radio Science Meeting. pp.1-4 (Feb. 2009)

12) A. Grzegorz, W. Wiesbeck and T. Zwick: "Differential feeding as a concept for the realization of broadband dual-polarized antennas with very high polarization purity", 2009 IEEE Antennas and Propagation Society International Symposium APSURSI'09, pp.1-4 (June 2009) 

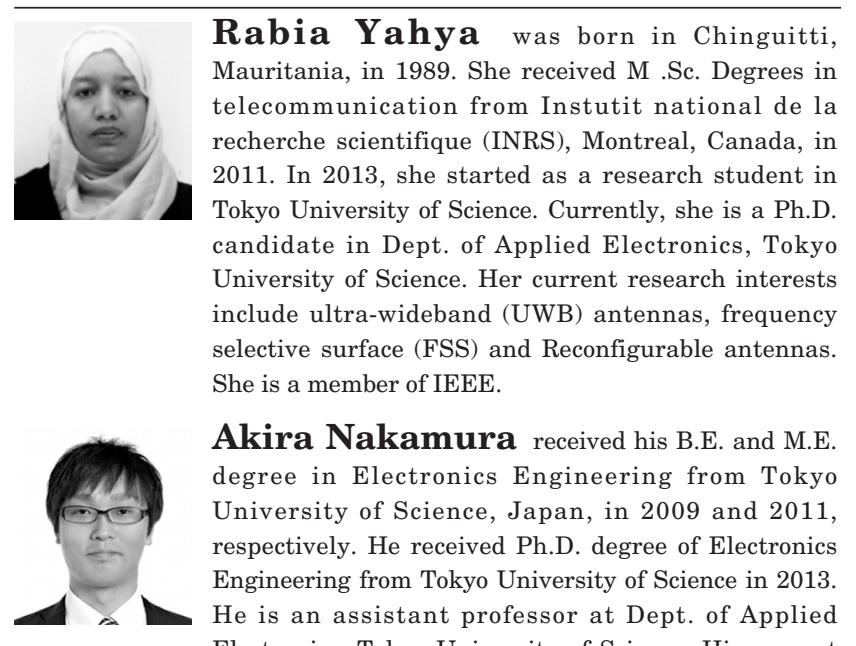

Akira Nakamura received his B.E. and M.E. degree in Electronics Engineering from Tokyo University of Science, Japan, in 2009 and 2011, respectively. He received Ph.D. degree of Electronics Engineering from Tokyo University of Science in 2013. $\mathrm{He}$ is an assistant professor at Dept. of Applied Electronics, Tokyo University of Science. His current research interests include wireless communication and broadcasting using OFDM modulation. He is a member of ITE, IEICE and IEEE.

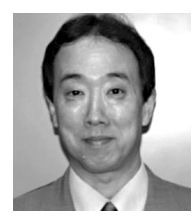

Makoto Itami received B. Eng., M. Eng. and Dr Eng. degrees from the University of Tokyo, in 1984, 1986 and 1989 , respectively. In 1989 , he joined the Department of Applied Electronics at Tokyo University of Science, Japan, and since 2008. He has been a Professor. His research interests are in the fields of communication systems and digital signal processing. $\mathrm{He}$ is a member of ITE, IEICE and IEEE.

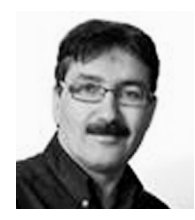

Tayeb A. Denidni (SM'09) received M. Sc. and $\mathrm{Ph} . \mathrm{D}$. degrees in electrical engineering from Laval University, Quebec City, QC, Canada, in 1990 and 1994, respectively. From 1994 to 2000, he was a Professor with the engineering department, Universit_ du Quebec in Rimouski (UQAR), Rimouski, QC, Canada, where he founded the Telecommunications laboratory. Since August 2000, he has been with the Institut National de la Recherche Scientifique (INRS), Universit_du Quebec, Montreal, QC, Canada. He found RF laboratory at INRS-EMT, Montreal. He has a great experience with antenna design and he is leading a large research group consisting of three research scientists, six Ph. D students, and one M.Sc. student. He served as a principal investigator on many research project sponsored By NSERC, FCI and numerous industries. His current research areas of interest include reconfigurable antennas using EBG and FSS structures, dielectric resonator antennas, metamaterial antennas, adaptive arrays, switched multi-beam antenna arrays, ultra-wideband antennas, microwave and development for wireless communications systems. From 2008 to 2010, Dr. Denidni served as an Associate Editor for IEEE Transactions on Antennas Propagation. From 2005 to 2007, Dr. Denidni has served as an Associate Editor for IEEE Antennas Wireless Propagation Letters. Since November 2015, Dr. Denidni has served as an Associate Editor for IET Electronics Letters. Since 2004, he has been elevated to the grade of Senior Member of the IEEE. In 2012 and 2013, he was awarded by INRS for outstanding research and teaching achievements. 